The review found little empirical evidence that HCAIs are managed in hospices, and concluded that the management of HCAIs in hospices is a misnomer. The broad purpose of this study was not to judge infection prevention and control practices in hospices, but to trigger thought and debate, and to develop collaborative approaches to HCAIs.

The study recommends further qualitative research based on patient perceptions, on the impact of HCAIs, and on infection prevention and control strategies. This knowledge could be used to inform strategies and interventions for palliative care patients.

\section{P-113 SERVICE INNOVATION TO MEET THE INCREASING DEMAND FOR DIETETICS IN PALLIATIVE CARE}

Anne Holdoway, Kathryn King, Ruth Gretton. Dorothy House Hospice Care, Bradford on Avon, UK

\subsection{6/bmjspcare-2016-001245.136}

Background Many life-limiting conditions negatively impact on an individual's ability to eat and drink pre-disposing an individual to malnutrition including its adverse consequences. Enjoyment and pleasure from eating and drinking can decline and diet becomes a source of psychological stress and anxiety. Although there exists a sound clinical rationale for dietetic interventions to optimise nutrition and alleviate symptoms dietetic services to hospices remains limited. In our own setting a specialist dietetic post (0.23 w.t.e.) was established in 2012. The service evolved to offer:

- a nutrition component to the patient rehabilitation and carer courses

- one to one consultations for patients with complex conditions requiring specialist dietary advice

- education and training for staff (internal and external).

Demand quickly out-stripped provision and therefore a creative, cost-effective solution was sought to continue to support patients and carers.

Aim To meet growing demand for dietetics with a newly created dietetic assistant post.

Results Following scoping and recruitment to the post, training was provided through case studies, supervision and e-learning. Since recruitment it has been possible to

- Further develop resources including a range of 'Handy Hints' (printed and web-based)

- Provide timely first-line dietetic interventions for patients and carers.

- Better support the specialist nurses.

- Streamline the use of oral nutritional supplements saving costs for the CCG.

A tool to identify patients requiring referral to the specialist dietitian for complex management has been validated and introduced.

Patient feedback in the form of patient evaluation and outcome measures have demonstrated the positive impact of the new service.

Conclusion To our knowledge this is the first hospice-based dietetic assistant post in the UK. Supervised by the specialist dietitian, this post has been better able to meet patient's needs. Similar posts across the UK would assist in meeting the growing demands of clients requiring supportive care who would benefit from diet modifications to maximise quality of life.

\section{P-114 A HOLISTIC APPROACH TO NUTRITION AND DIET IN PALLIATIVE CARE}

Jill Souter, Agata Czerwinska, Sarah Cooley, Anne Holdoway. Dorothy House Hospice Care, Bradford on Avon, UK

10.1136/bmjspcare-2016-001245.137

Background In response to the Care Quality Commission and NHS contract requirements, a validated screening tool; the Malnutrition Universal Screening Tool 'MUST' was introduced into our hospice setting in 2012. Subsequent research amongst nursing staff confirmed lack of confidence in 'MUST' in identifying and guiding decisions for appropriate nutritional care. A project team was established to investigate how we might achieve appropriate nutritional care taking into account the changing and complex needs of patients and carers in the hospice setting.

Aims To identify nutritional issues experienced and develop a nutrition assessment tool to sensitively determine and deliver nutritional care to meet the needs of patients and carers.

Methods A practice development approach was utilised engaging all stakeholders. The approach included:

- questionnaires to staff

- discussion forums involving members of the healthcare team, patients, carers and catering.

- qualitative interviews with patients/carers.

Findings were used to initiate change and refine and validate a nutrition assessment tool.

Results Results from the questionnaires and forums identified issues affecting the delivery of optimal nutritional care. Solutions were identified and tested, patient and carer participation was a key component. A new approach to menu planning, including 13-day cycles, menu choices and portion size was developed. A new nutrition assessment tool - the 'Patient Led Assessment for Nutritional Care' (PLANC) was developed, tested, refined and validated to identify nutritional issues and determine care taking into account the stage of disease. Nutrition and hydration were integrated into the shared patient record. New pathways and resources were created to address common nutritional problems with triggers for escalation management to a specialist dietitian and dietetic assistant when required.

Conclusion The practice development process for change fitted well with the ethos of the organisation and resulted in practice improvements to enhance care. The 'PLANC' tool for nutrition assessment has been integrated into care across the settings.

\section{P-115 A HOSPICE BREATHLESSNESS MANAGEMENT INTERVENTION SERVICE - A BLEND OF THE OLD WITH THE NEW!}

${ }^{1,2}$ Declan Cawley, ${ }^{1}$ Clare Horne. ${ }^{1}$ Pilgrims Hospices, Canterbury, UK; ${ }^{2}$ University Of Kent

\subsection{6/bmjspcare-2016-001245.138}

Background Breathlessness is one of the most distressing symptoms reported in the literature with the prevalence rising with the vast multi-morbidity people are living with. The breathlessness management intervention for patients with a variety of mixed pathologies but they have the commonality of refractory, end stage breathlessness. The approach employs non-pharmacological approaches but clearly can be in tandem to pharmacological agents. 